\title{
Mucocele formation after frontal sinus obliteration*
}

Fleur S. Hansen', Nicolien A. van der Poel², Nicole J.M. Freling33, Wytske J. Fokkens ${ }^{2}$

' Dept. of Otorhinolaryngology, Central Military Hospital, Utrecht, The Netherlands

2Dept. of Otorhinolaryngology, Academic Medical Centre, Amsterdam, The Netherlands

${ }^{3}$ Dept. of Radiology, Academic Medical Centre, Amsterdam, The Netherlands
Rhinology 56; 2: 106-110, 2018

https://doi.org/10.4193/Rhin17.187

*Received for publication:

August 19, 2017

Accepted: January 7, 2018

Background: A possible complication of frontal sinus obliteration with fat is the formation of mucoceles. We studied the prevalence of mucoceles as well as and the need for revision surgery.

Methods: Retrospective case review of forty consecutive patients undergoing frontal sinus obliteration from September 1995 to February 2012 for chronic rhinosinusitis (26), frontal mucocele (12) or frontal osteoma (2) with an average follow up of 80 months (range 15-218). MRI of the paranasal sinuses was performed in all. Outcome measures included MRI signs of mucocele formation in the obliterated frontal sinus, revision surgery, symptom burden.

Results: Magnetic resonance imaging (MRI) showed potential postoperative frontal sinus mucoceles in $6 / 40$ patients. In 3 patients (7.5\%) a revision operation was performed, revealing mucoceles in two cases. A wait and scan-policy in the other 3 patients confirmed the presence of a mucocele in 1 of these patients. The majority of patients (33/40, 83\%) was asymptomatic at the last follow up.

Conclusion: The prevalence of mucoceles and revision rate in this series was 7.5\% (3/40). MRI can improve detection rate and reduce / avoid unnecessary revision surgery after frontal sinus obliteration.

Keywords: Frontal sinus obliteration, osteoplastic flap, chronic rhinosinusitis, mucocele, MRI

\section{Introduction}

The surgical management of the frontal sinus with its complex and variable drainage path remains one of the greatest challenges in otolaryngology ${ }^{(1)}$. The frontal sinus can be approached endonasally (by for instance a modified endoscopic Lothrop or Draf III-procedure) or externally using the osteoplastic flap technique with or without obliteration ${ }^{(2-5)}$. Technical improvements like curved drills and topical mitomycin-c application have broadened the possibilities of endonasal frontal sinus surgery ${ }^{(6-8)}$. However, in some cases the external approach and obliteration of the frontal sinus is unavoidable. In the Academic Medical Centre, a tertiary referral centre, external frontal sinus surgery is reserved for symptomatic patients who have failed maximum medical therapy as well as functional endoscopic frontal sinus procedures and/or Draf III-procedure(s) and for patients with tumours in the frontal sinus, which are inaccessible transnasally.
In general and in dialogue with the patient, 2-3 revision Draf IIIprocedures are tried before we decide to do an external frontal approach. Incapability of maintaining adequate endonasal drainage of the frontal sinus after endoscopic surgery is found to be more common in patients with allergy ${ }^{(6,10)}$ and/or a narrow neo-ostium ${ }^{(10,11)}$. Endonasal drainage of the frontal sinus is not more likely to persist after external frontal sinus surgery than after endonasal surgery such as Draf III. Therefore, we advocate combining the external approach with occlusion of the frontal recess and obliteration of the frontal sinus with abdominal fat after removal of all frontal sinus mucosa.

Patient satisfaction after frontal sinus obliteration is generally good ${ }^{(12-15)}$, but a medium to long term complication often described in the literature is development of mucoceles in the obliterated frontal sinus with incidences as high as $23 \%^{(16-18)}$. Mucoceles can cause destruction of neighboring bony structures 
by expansive growth, creating a need for surgical re-exploration. In this study we investigate the prevalence of mucoceles after frontal sinus obliteration.

\section{Materials and methods}

This is a retrospective case study of all patients undergoing a frontal sinus obliteration procedure in our institution, from September 1995 to February 2012. Only patients with a follow up equal or greater than 15 months were included. Indications for surgery, demographic patient data, medical history including smoking, allergy, asthma or ASA triad and surgical history were recorded.

All patients underwent preoperative spiral computed tomography $(\mathrm{CT})$ scans, with axial plane parallel to the hard palate, and images reconstructed in coronal and sagittal planes. Magnetic resonance imaging (MRI)-scans focusing on mucoceles within the frontal sinus were performed yearly during the first five years postoperatively and then every $2-3$ years, in most patients until 10 years postoperatively. SET2 axial, coronal and sagittal, axial SET1 with and without fatsat, 3mm slice thickness, 512×256 resolution matrix, 200 FOV (Avanto 76x18, 1.5T. Siemens, Erlangen, Germany). All MRI-scans were reviewed by two otorhinolaryngologists and a specialist head and neck radiologist. We defined mucoceles as expansive, well-defined, rounded-to-oval lesions within the frontal sinus, connected to the sinus wall with or without bone remodelling (see Box 1 for radiological criteria). Approval by an ethics committee was not requested.

\section{Technical aspects of frontal sinus obliteration}

After coronal incision or incision in a forehead fold a scalp flap is developed up to the supraorbital rim with preservation of the supraorbital and supratrochlear nerves. The frontal sinus is marked using navigation or with an occipitofrontal 1:1 x-ray template. The frontal sinus is openend with saw and chisel, preparing a bevelled anterior wall of the frontal sinus that can be placed back. If the frontal wall is absent or for other reasons cannot be used a reconstruction of the anterior wall with titanium mesh is performed. An elevator is used to circumferentially elevate the mucosa in the frontal recess bilaterally. Subsequently the whole frontal sinus is drilled with sharp and diamond drills of various sizes $(1-6 \mathrm{~mm})$ to meticulously remove all the sinus mucosa, the periost and a thin layer of bone from the entire sinus surface. All septations are drilled down to generate a single smooth sinus cavity. Extra attention is given to the supraorbital region and the superior rim of the frontal sinus. When the surgeon is convinced that everything is drilled perfectly, a second round of drilling is performed. The mucosa in the frontal recess, or the neo-ostium (in case of previous Draf III), is then inverted and pushed inferiorly. A piece of bone from the outer layer of calvarian bone is harvested somewhere from the exposed skull. The piece is exactly modulated to wedge into the frontal sinus

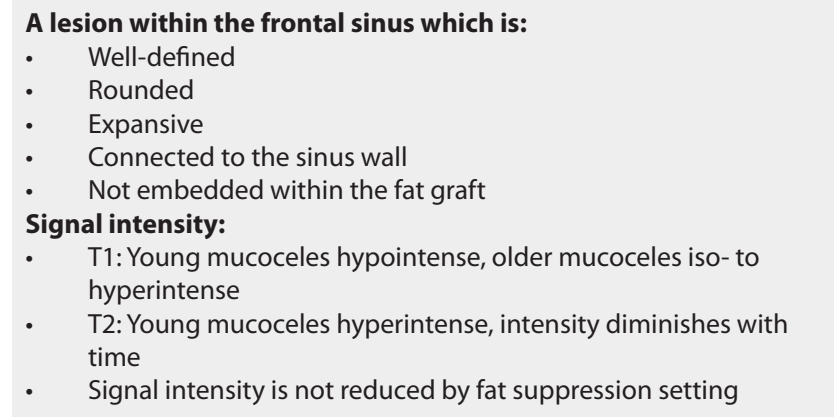

Box 1. Radiologic criteria for diagnosis of clinically relevant mucocele after frontal sinus obliteration.

recess or the neo-ostium (after DRAF III). Finally the piece of bone is fixed with bone wax. The frontal sinus is filled with fat. Clinical outcome was based on the presence of symptoms as reported by the patient at last follow up.

\section{Results}

Patient characteristics

Forty patients were included in this study ( 23 males) with a mean age of 50 (range 17 to 76 years). Twenty-six patients had chronic frontal rhinosinusitis: 17 had chronic rhinosinusitis without nasal polyps (CRSsNP), 9 had chronic rhinosinusitis with nasal polyps (CRSwNP). Twelve patients had mucoceles and 2 had osteomata of the frontal sinus (Table 1). Ten patients (25\%) had skin-prick test-confirmed allergy and 12 patients had asthma. Four patients had aspirin-sensitive asthma and nasal polyps (ASA triad).

Thirty-four patients underwent frontal sinus obliteration as a single procedure. In five patients endonasal surgery was done in the same setting and in one patient the frontal sinus was cranialized before obliteration. This was a young male with type II neurofibromatosis. The posterior frontal sinus wall was largely destroyed. Therefore, it was decided to drill away the remains of the posterior wall, cranializing the sinus. The space between the anterior sinus wall and the brain was then obliterated with abdominal fat.

Four patients underwent frontal sinus obliteration as a primary procedure, all because of extensive frontal mucoceles with large defects in the orbital roof and in the posterior wall of the frontal sinus and a very small anteroposterior distance between the anterior nasal beak and the skull base. Thirty-six patients had had previous sinus surgery. On average patients had undergone 4.2 previous procedures (maximum 11). Thirty-two patients (80\%) had had previous endoscopic surgery, ten had undergone a Draf III-procedure (25\%) and eighteen (45\%) had had external frontal sinus procedures without obliteration.

Mean follow up was 80 months, range 15 to 218 months. Median follow up was 70 months. 
Table 1. Indications for surgery and revision rates.

\begin{tabular}{|c|c|}
\hline Indication & $\mathbf{N}$ \\
\hline CRSsNP & 16 \\
\hline Revision surgery & 2 \\
\hline CRSwNP & 9 \\
\hline Mucocele & 9 \\
\hline Revision surgery & 1 \\
\hline CRSsNP + mucocele & 3 \\
\hline CRSsNP + CF & 1 \\
\hline CRSsNP + osteoma & 1 \\
\hline Gardner's syndrome & 1 \\
\hline
\end{tabular}

\section{Postoperative mucoceles}

We reviewed $141 \mathrm{MRI}$-scans of 40 patients. In 45 original radiology reports regarding 21 patients a possibility of a mucocele was reported. In 15 patients careful review by three of the authors (FH, NF, WF) revealed an absence of growth and/or bony destruction over time. It was therefore concluded that these lesions were non-growing mucoceles or not mucoceles at all. Most of these previously alleged mucoceles were re-labeled as residual supra-orbital cells. Abnormalities surrounded by fat and/or without contact with a sinus wall were also considered not to be mucoceles. In the end, postoperative MRI-scans showed abnormalities which were radiologically suspected to be mucoceles in 6 patients. In three of these patients a wait and scan-policy was adopted. In two patients no measurable progression was found in the 3-4 years they were monitored until now. In the third patient the abnormality grew $2-5 \mathrm{~mm}$ in 6 years. Because of the absence of growth we concluded the first two lesions were not likely to be mucoceles after all, but further evaluation over time is necessary for a definitive conclusion. Three patients underwent revision surgery 10 months, 19 months and 7 years after the obliteration. Revision surgery revealed frontal mucoceles in two patients: one in the obliterated frontal sinus and one within frontal cells that were overlooked during the original obliteration. In the third patient, operated seven years after the primary procedure, the frontal sinus contained no mucoceles, but inflamed residual mucosa. Repeated MRI-scans in the 3 to 6 years after revision surgery did not reveal recurrent mucoceles in any of the 3 revision patients.

In this series the incidence of mucocele formation was $7.5 \%$ $(3 / 40)$.

\section{Clinical outcomes}

At the end of the follow up period the majority of patients ( $n=33,82.5 \%$ ) was asymptomatic, while the remaining seven patients reported bothersome symptoms, described as either frontal pressure [5] or frontal headache [2]. All of these symptomatic patients had a diagnosis of chronic rhinosinusitis and had all previously failed multiple endoscopic sinus procedures, while five of them had also failed at least one external procedure prior to frontal sinus obliteration. We think most of these patients had non-sinugenic facial pain, but osteitis cannot be excluded. Two of these 7 patients had a mucocele on MRI after frontal sinus obliteration. One underwent revision obliteration, but remained symptomatic despite this.

\section{Discussion}

The data from this retrospective case review with well known limitations suggest a prevalence of postoperative mucoceles of $7.5 \%$, which is comparable to the $9.8 \%$ described by Weber et al. ${ }^{(16)}$, but notably lower than the prevalence of $23 \%$ found by Loevner et al. ${ }^{(17)}$. What causes this discrepancy? Was the follow up period too short? Scangas et al. showed that mucoceles can occur as long as 18 years after open frontal sinus surgery ${ }^{(19)}$. This corresponds with the longest follow up of 218 months in our study, but 33 patients had a follow up of less than 10 years at the moment of writing this article. However, the mucoceles we found were detected within the first two postoperative years. Weber et al. describe a follow up period of 1 to 12 years and found mucoceles 11 to 130 months postoperatively. Loevner studied 13 patients, of which 10 were followed up 14 months or less. No mucoceles were detected in this group. The other three patients had a follow up of 32 months, 9 years and 12 years and these were the patients in which mucoceles were found. The authors acknowledge the need for a long follow up period and will continue to monitor the patients in this study. A difference in surgical technique could be a cause of the difference in mucocele rates, but a difference in criteria on which a lesion is labeled a mucocele on MRI is a more likely reason.

Because the decision on reintervention can be purely based on MRI findings, an adequate radiological definition of mucoceles and a differentiation from the fat graft is of utter importance. Signal intensity of mucoceles on $\mathrm{T} 1$ and $\mathrm{T} 2$ is dependent upon the viscosity and the fluid content. On T1 mucoceles have low signal intensity initially, but a more dehydrated mucocele changes from isointense to hyperintense. On T2, mucoceles are hyperintense owing to their high water content. With time, the intensity may diminish due to water absorption and increased protein concentration ${ }^{(20,21)}$.

The density of the fat graft is variable between patients and over time. This might be caused by the formation of fibrosis and/or haemorrhage within the graft ${ }^{(22)}$. In determining whether tissue seen in the frontal sinus is fat or not, applying the fat suppression technique is helpful (Figure 1).

Not all round lesions with the signal intensity of mucoceles are indeed mucoceles. In some cases the fat graft takes the typical round shape of a mucocele because a bony concavity was 


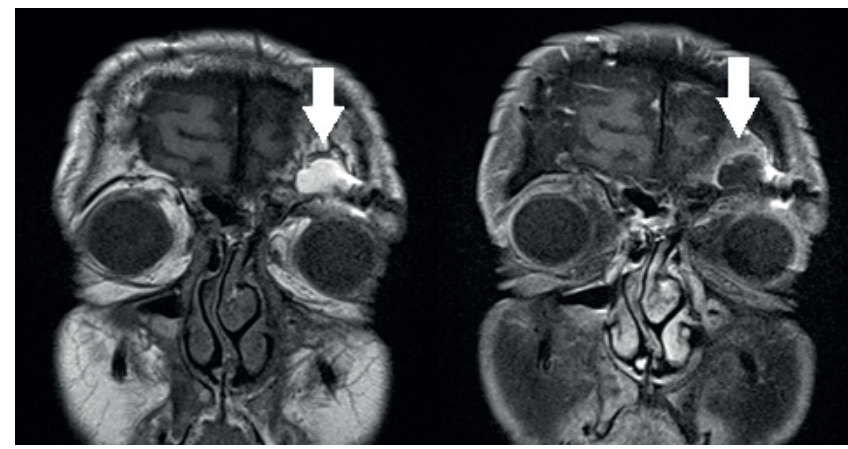

Figure 1. On the left a T1 image with a supraorbital abnormality. On the right the same abnormality in a T1 with fat suppression-setting. Signal intensity is markedly lower in fatsuppression-setting and therefore we can conclude that this tissue is fat.

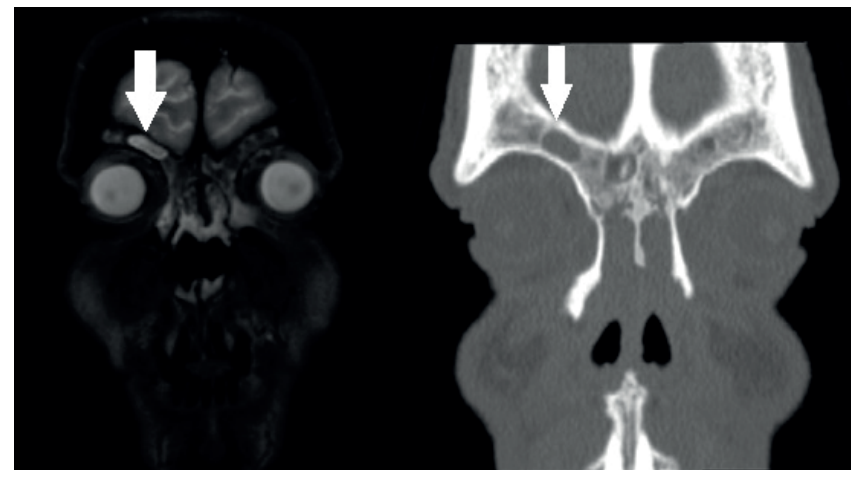

Figure 2. At first glance a mucocele, but after closer examination fat taking the shape of a mucocele on postoperative MRI due to preoperative concavity as seen on preoperative CT.

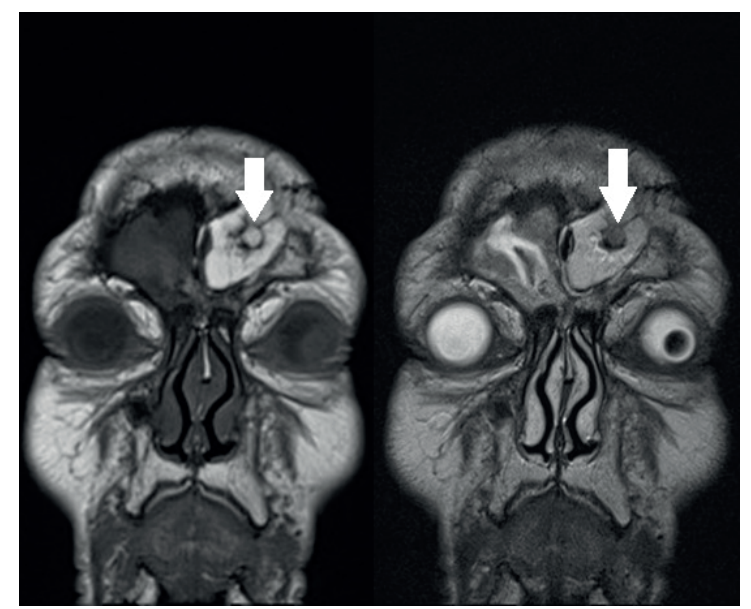

Figure 3. T1 and T2-image of lesion surrounded by abdominal fat. Since the fat graft does not contain mucosa, this lesion cannot be a mucocele.

present in that location before obliteration, perhaps due to a mucocele. Comparison of pre-operative CT-scans and postoperative MRI-scans is very informative in these cases (Figure

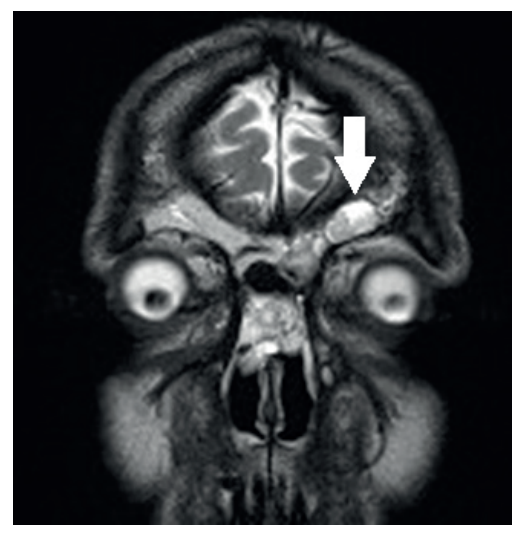

Figure 4. Mucus filled supraorbital cell on postoperative MRI.

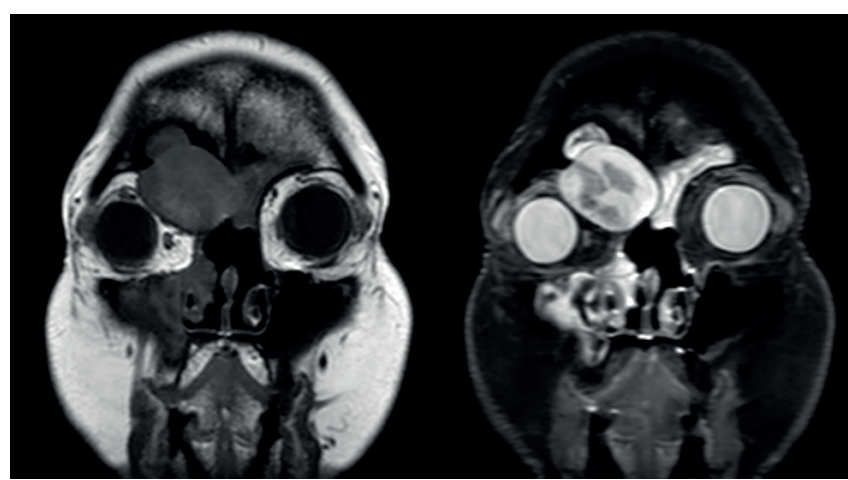

Figure 5. T1 and T2-image of a large frontal mucocele.

2). An anomaly which is not attached to the sinus wall, but is fully contained within the inserted fat is very unlikely to be a mucocele, since the fat graft does not contain mucosa (Figure 3). Non-growing lesions can be mucus-filled pre-existing cavities (Figure 4). These can be located above or under the level of occlusion of the obliterated frontal sinus, for instance in the frontal recess. These cells, although opacified, probably drain into the ethmoid and thus do not cause problems. Revision rate in our series was $7.5 \%$. The revision rate found in this series is lower than in series studying endonasal radical frontal sinus surgery, like Draf III-procedure: 32\% by Georgalas ${ }^{(6)}$ and Schlosser ${ }^{(23)}, 15 \%$ by Samaha ${ }^{(24)}$ and $13 \%$ by $\operatorname{Tran}^{(25)}$. However, we certainly do not advocate to do obliteration instead of Draf III. The significant higher morbidity and the need for long term MRI control make obliteration last resort surgery.

\section{Conclusion}

The prevalence of mucoceles and revision rate in this series were $7.5 \%$ (3/40). MRI is well suited to detect mucoceles during follow-up of patients after FSO. The application of well-defined radiological criteria can reduce unnecessary revision surgery as well as multidisciplinary discussions between radiologists and otorhinolaryngologists to fully understand the differential diagnosis. Clinical outcome was favourable in 83\% (33/40). 


\section{Authorship contribution}

FSH: Data collection, data analysis, writing paper; NAvdP:

Revision paper; NJMF: Data collection (MRI), revision paper; WJF:

Data collection, supervision data analysis, revision paper.

\section{Conflict of interest}

Financial support or funding: none

Conflicts of interest: none

\section{References}

1. Lai WS, Yang PL, Lee $\mathrm{CH}$, et al. The association of frontal recess anatomy and mucosa disease on the presence of chronic frontal sinusitis: a computed tomographic analysis. Rhinology. 2014 Sep;52(3):208-14.

2. Anand VK, Hiltzik DH, Kacker A, Honrado C. Osteoplastic flap for frontal sinus obliteration in the era of image-guided endoscopic sinus surgery. Am J Rhinol. 2005;19(4):40610.

3. Schlosser RJ, Zachmann G, Harrison S, Gross CW. The endoscopic modified Lothrop: long-term follow-up on 44 patients. Am J Rhinol. 2002;16(2):103-8

4. Ulualp SO, Carlson TK, Toohill RJ. Osteoplastic flap versus modified endoscopic Lothrop procedure in patients with frontal sinus disease. Am J Rhinol. 2000;14(1):21-6.

5. Sama A, McClelland L, Constable J. Frontal sinus mucocoeles: new algorithm forsurgical management. Rhinology. 2014 Sep:52(3):267-75.

6. Georgalas C, Hansen F, Videler WJ, Fokkens WJ. Long term results of Draf type ॥ (modified endoscopic Lothrop) frontal sinus drainage procedure in 122 patients: a single centre experience. Rhinology. 2011:49(2):195.

7. Konstantinidis I, Chatziavramidis A, Constantinidis J. A novel technique for mitomycin-c application in frontal sinus surgery. Rhinology. 2014 Sep;52(3):276-80.

8. Samaha M, Cosenza MJ, Metson R. Endoscopic frontal sinus drillout in 100 patients. Arch Otolaryngol Head Neck Surg. 2003;129(8):854-8.

9. Georgalas C, Fokkens WJ. Rhinology and Skull Base Surgery. From the Lab to the Operating Room: an Evidence-based Approach. Stuttgart: Thieme, 2013.

10. Casiano RR, Livingston JA. Endoscopic
Lothrop procedure: the University of Miami experience. Am J Rhinol. 1998;12(5):335-9.

11. Tran KN, Beule AG, Singal D, Wormald PJ. Frontal ostium restenosis after the endoscopic modified Lothrop procedure. Laryngoscope. 2007;117(8):1457.

12. Kristin J, Betz CS, Stelter K, Berghaus A, Leunig A. Frontal sinus obliteration-a successful treatment option in patients with endoscopically inaccessible frontal mucoceles. Rhinology. 2008;46(1):70-4.

13. Hardy JM, Montgomery WW. Osteoplastic frontal sinusotomy: an analysis of 250 operations. Ann Otol Rhinol Laryngol. 1976;85(41):523-32.

14. Correa AJ, Duncavage JA, Fortune DS, Reinisch L. Osteoplastic flap for obliteration of the frontal sinus : five years' experience. Otolaryngol Head Neck Surg. 1999;121(6):731

15. Mendians AE, Marks SC. Outcome of frontal sinus obliteration. Laryngoscope. 1999;109(9):1495.

16. Weber R, Draf W, Keerl R, et al. Osteoplastic frontal sinus surgery with fat obliteration: technique and long-term results using magnetic resonance imaging in 82 operations. Laryngoscope. 2000;110(6):1037.

17. Loevner LA, Yousem DM, Lanza DC, Kennedy DW, Goldberg AN. MR evaluation of frontal sinus osteoplastic flaps with autogenous fat grafts. Am J Neuroradiol. 1995;16(8):1721-6.

18. Silverman JB, Gray ST, Busaba NY. Role of osteoplastic frontal sinus obliteration in the era of endoscopic sinus surgery. Int J Otolaryngol. 2012;2012:501896.

19. Scangas GA, Gudis DA, Kennedy DW. The natural history and clinical characteristics of paranasal sinus mucoceles: a clinical review. Int Forum Allergy Rhinol. 2013 Sep;3(9):7127

20. Van Tassel $P$, Lee $Y Y$, Jing BS, De Pena
CA. Mucoceles of the paranasal sinuses: MR imaging with CT correlation. Am J Roentgenol. 1989;153(2):407-12.

21. Capra GG, Carbone PN, Mullin DP. Paranasal sinus mucocele. Head Neck Pathol. 2012;6(3):369-72.

22. Catalano PJ, Lawson W, Som P, Biller HF. Radiographic evaluation and diagnosis of the failed frontal osteoplastic flap with fat obliteration. Otolaryngol Head Neck Surg. 1991;104(2):225-34.

23. Schlosser RJ, Zachmann G, Harrison S, Gross $\mathrm{CW}$. The endoscopic modified Lothrop: long-term follow-up on 44 patients. Am J Rhinol. 2002; 16: 103-108.

24. Samaha M, Cosenza MJ, Metson R. Endoscopic frontal sinus drill- out in 100 patients. Arch Otolaryngol Head Neck Surg. 2003; 129: 854-858.

25. Tran KN, Beule AG, Singal D, Wormald PJ. Frontal ostium restenosis after the endoscopic modified Lothrop procedure. Laryngoscope. 2007; 117: 1457-1462.

Fleur S. Hansen, MD PhD

Dept. of Otorhinolaryngology

Central Military Hospital

Lundlaan 1

3584 EZ Utrecht

The Netherlands

Tel: $+31-30-2502210$

E-mail:

fs.besseling.hansen@mindef.nl 